\title{
An Online Non-Invasive Condition Monitoring Method for Stepping Motor CRDM in HTGR
}

\author{
S. Bakhri ${ }^{1 *}$ and N. Ertugrul ${ }^{2}$ \\ ${ }^{1}$ Center for Nuclear Reactor Technology and Safety, National Nuclear Energy Agency \\ Puspiptek Area, Serpong, Tangerang 15310, Indonesia \\ ${ }^{2}$ School of EEE, The University of Adelaide, Adelaide, 5005, Australia
}

\section{ARTICLE INFO}

Article history:

Received 19 November 2014

Received in revised form 13 April 2016

Accepted 22 June 2016

\section{Keywords:}

CRDM

Condition monitoring

Stepper motor

HTGR

\begin{abstract}
A B S T R A C T
Control Rod Drive Mechanism (CRDM) based on stepping motor is one of the components applied in High Temperature Gas Coold Reactor (HTGR) to control the reactivity as well as to maintain the safety of reactor. The stepping motor requires a unique condition monitoring to avoid any failures especially due to the specific environments of CRDM in HTGR such as the allowable of high temperature, high radiation and the location of stepper motor inside a pressure shell. This research aims to demonstrate an online non-invasive condition monitoring method without direct access to the CRDM of HTGR based on voltage and stator current measurements. A simple stepping motor CRDM simulator is employed. The online condition monitoring is carried out by direct pattern matching of the output signals of logic generator block and the output signals of motor driver. The online method utilizes signature patterns of voltage and stator current signals of the healthy motor as a baseline for healthy motor. In addition, the method is applied to detect highresistance problem on the connector between the motor driver block and the stepper motor to show the effectiveness and the applicability of this method. The online condition monitoring system demonstrates a capability to identify a minimum detectable simulated high-resistance for about $2.9 \%$ which decreases the measured stator current and motor's torque for around $5.1 \%$ and $3.3 \%$, respectively. The paper also points out signatures of healthy motor, including mutual inductions of the motor's winding in voltage and current measurement which can be used as the fault symptom indicators for online monitoring purposes.
\end{abstract}

\section{INTRODUCTION}

The reactivity control is necessary to maintain the safety of High Temperature Gas Coold Reactor (HTGR) power plant during the normal as well as the emergency conditions by inserting or withdrawing the control rod using an arrangement known as Control Rod Drive Mechanism (CRDM). To avoid the failure of CRDM, it requires not only a reliable drive mechanism but also a method to understand the symptom of the CRDM degradation which can be identified through a condition monitoring technique. It should be noted here that in

\footnotetext{
* Corresponding author.

E-mail address: bakhrisy@batan.go.id

DOI: http://dx.doi.org/10.17146/aij.2016.516
}

nuclear power plant, the control rod may involve various type of drive mechanisms such as magnetic coils [1], hydraulic [2] as well as the electric motor drive [3-5].

Recent development of the CRDM in HTGR can be found in the new reactor such as HTR-10 (10MWth), HTTR-30 (30MWth) and HTR-PM (200MWth). Issues of the research and development in CRDM HTGR mainly focus on the design and technique to overcome the effect of high temperature, helium environment, as well as high radiation exposure. Research in [6] shows that temperature and radiation field inside the pressure shells containing CRDM of HTR-10 may reach $150^{\circ} \mathrm{C}$ and $7.61 \mathrm{~Sv} h-1$, respectively. In case of HTTR-30, the maximum allowable temperature 
services inside the standpipe of CRDM was maintained below $75^{\circ} \mathrm{C}$ [7] by indirect air cooling outside the standpipes as well as by injecting small amount cold Helium known as purge gas through the standpipes. In case of HTR-PM, the temperature ambient of the CRDM under long-term radiation exposure is $150-250^{\circ} \mathrm{C}$ [8]. Because of these ambient conditions, electric motor type of CRDM is more suitable than the magnetic coils, pneumatic, or hydraulic drive.

The current status of electric motor based CRDM in HTGR is that the stepping motor is employed for the HTR-10 [6] and HTR-PM [9], and the alternating current motor is for the HTTR-30 $[7,10]$. The stepper motor has been identified to have a better safety due to the omission of traditional counterweight or brake clutch $[6,8]$. The stepper motor also has high accuracy, fast response, simple control as well as simple kinematics which significantly contribute to its high reliability $[6,8,11]$. Therefore, this paper focuses on the stepper motor based CRDM.

Studies have been carried out in the literature to understand the characteristics of stepping motor which later might be applied for condition motoring purposes. For example, in case of the nuclear reactor applications, a study has produced an analytical model for the CRDM of VVER 1000 MW based on permeance magnetic model approach [12]. The model was aimed to understand the static and dynamic characteristic of linear stepper motor for CRDM, i.e. the electromagnetic force of the stepper motor due to the armature speed and the effect of displacement during the various mode operations [12]. Another study demonstrated more advance model by adding electromechanical resonances simulation which may decrease the performance due to the vibrations, stalling, or losing steps $[13,14]$. In [9], a dynamic stepping motor model it was also proposed to combine with nonlinear dynamic Modular High Temperature Reactor (MHTGR). Furthermore, the characteristics of the control of motor stepper simulator for CRDM using Programmable Logic Controller (PLC) to simulate normal operation of CRDM based with wave drive and half step is studied in [15].

However, these previous cited works have not studied the implementation of online condition monitoring system of stepping motor of the CRDM especially in HTGR. In $[13,14]$, the model was used for the stepper motor control system and the parameter identification purposes only, not used for the online condition monitoring with the capability to identify symptom of faults or component degradations. Furthermore, the previous stepper motor based CRDM in PWR [12], has not considered the condition monitoring. An effective model in such system, should consider the degradations in system as well as the potential faults. In addition, the previous works in $[6,9,15]$ only emphasize the control of CRDM not consider for further implementation of fault detection and online monitoring.

Therefore, this study aims to address these challenges by demonstrating a non-invasive online monitoring method which can be applied for the CRDM in HTGR. This method provides the assessments of CRDM to identify normal, degradations as well as faults without stopping the control rod control system in continuous manner and without direct access to the motor. This method will be beneficial for wide range of stepper motor applications even though the HTGR application is the focus in this paper.

This paper describes the proposed online noninvasive method to monitor the condition of stepping motor CRDM in HTGR. The online noninvasive method consists of online acquisition of the output electric signals of each block component of the CRDM, followed by signal pattern matching based on its shape and timing between each block. The key of this online monitoring method is to identify the signature differences of the measured supply voltage, electric current signals including the produced torque between the healthy and faulty motor. To see the effectiveness and the applicability of the proposed method, a case study on the simulated asymmetrical supply problems is provided. The asymmetrical supply problems can be caused by high-resistance connections between the circuit driver and the electric motor [16,17]. It was pointed out in [18-20] that the high-resistance connections can be produced by contact surface contamination, corrosion/oxidation of the connectors, pitted/worn/rough connector surface, under/overtightening of connectors, mismatch between connectors, or looseness of connectors due to vibration and thermal cycling.

After the theory section in this paper, the experimental arrangement of a simple CRDM stepping motor based simulator which consist of a motor stepper which is driven by a logic generator (PLC) and a motor driver (H-Bridge) including the applied control pattern is presented. The online monitoring system is also described to show the simplicity of this method. In results and discussions section, the effectiveness of the online monitoring approaches is demonstrated. The online condition monitoring of the logic pattern generator is studied, followed by the stepper motor driver block analysis. Various voltage and electric current signatures of the healthy motor under different control logic 
patterns are also identified as the baseline information. A case example in online detection of motor's faults due to high-resistance problems based on measured voltage, stator current and torque is also investigated. These steps are carried out to ensure that the proposed online non-invasive method covers all components as well as to ensure the applicability of the method for condition monitoring purposes.

\section{THEORY}

\section{Control rod drive mechanism (CRDM)}

Figure 1 shows one of the examples of CRDM for the HTGR type power plant (HTR-10) [6]. Note that, the control rod drive mechanism has a stepping motor, a magnetic damper, and a gear box, a sprocket wheel, a worm-gear reducer and control rod position detection system. The stepper motor provides a rotating force to lower and withdrawn control rod at certain speed of rotation. The requirement of control rod insertion speed for the HTR-10 is $10 \mathrm{~mm} \mathrm{~s}^{-1}$ or equal with $30 \mathrm{rpm}$ with the emergency insertion less than 8s. Another component such as magnetic damper which requires no electricity to generate damping torque is employed to reduce the speed during the free fall emergency insertions for no more than $0.8 \mathrm{~m} \mathrm{~s}^{-1}$ and to minimize any shock energy at the end buffer of control rod. The above speed requirement is also implemented to accommodate the maximum allowable speed of the sprocket wheel. The gear box is used to reduce the speed from the $30 \mathrm{rpm}$ into $3 \mathrm{rpm}$ and also increase the applied torque. To measure the position of control rod, a worm-gear reducer is employed between the sprocket wheel device and the synchro. The total rotation of the synchro for $282^{\circ}$ indicates a whole stroke for the movement of control rod. Such CRDM is controlled by an arrangement which consist of a stepping motor driver, a pulse signal generator, a countertimer interface and a computer.

The control mechanism shown and explained above indicates that the condition monitoring should be non-invasive due to the limitations to the control system. The motor, gear, sprocket wheel and synchro also are located inside a pressure shell. The generated vibrations due to the operation of stepper motor, gear, wheel, chain and synchro also requires the vibrations to be monitored. One literature presented the possibility to monitor early failures during the manufacturing steps based on vibration monitoring [21]. However, the allowable maximum temperature of CRDM shells of HTGR up to $150^{\circ} \mathrm{C}$ [6] even higher in HTR-PM, i.e. $250^{\circ} \mathrm{C}$ [8], limits this vibration monitoring approaches due to the operating limits of the practical accelerometers. An indirect acoustic emissions using microphone also is not suitable since the upper pressure shell attenuates the sound propagations. In addition, the acoustic emission sensor (the contact or non-contact sensors) might not even provide better results compare with the vibration monitoring under the influence of the high temperature of upper pressure shell. These limitations requires an indirect monitoring technique such as using voltage and electric current analysis which is considered in this paper.

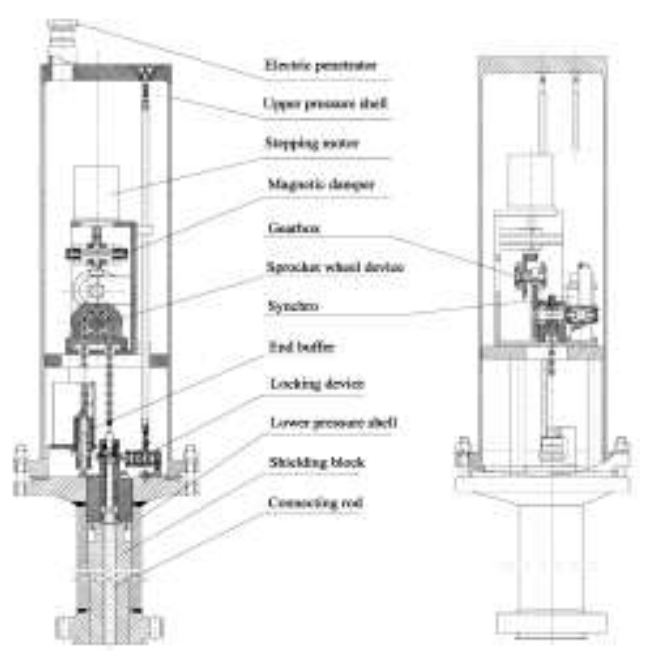

Fig. 1. The typical components of CRDM HTR-10 [6].

\section{Condition monitoring of CRDM}

The condition monitoring of control rod has been introduced considering several aspects. The comprehensive assessment in aging-related degradations of CRDM was mainly focused on Westinghouse type of PWR [22]. The Westinghouse PWRs employ grippers and magnetizing coils system which are different than the type of HTR-10 previously mentioned. However, it was found out that two important factors to be considered in CRDM for the condition monitoring purposes, i.e. failure distributions and fault monitoring system.

The common faults and failures in CRDM, and the causes of failures have been investigated and summarized in Fig. 2 (a). As it is shown in the figure, power and logic systems are the major contributors of the failures, followed by rod position indicator and cable/connector. It should be noted here that these components (logic and power supply system) are also installed in the HTGR types, which confirms the necessity of condition monitoring of these systems. 


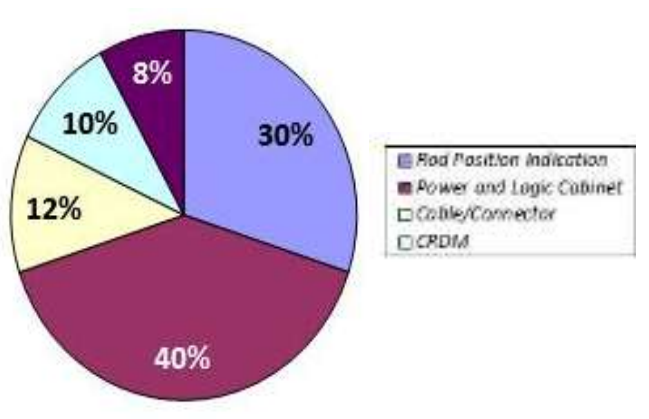

(a)

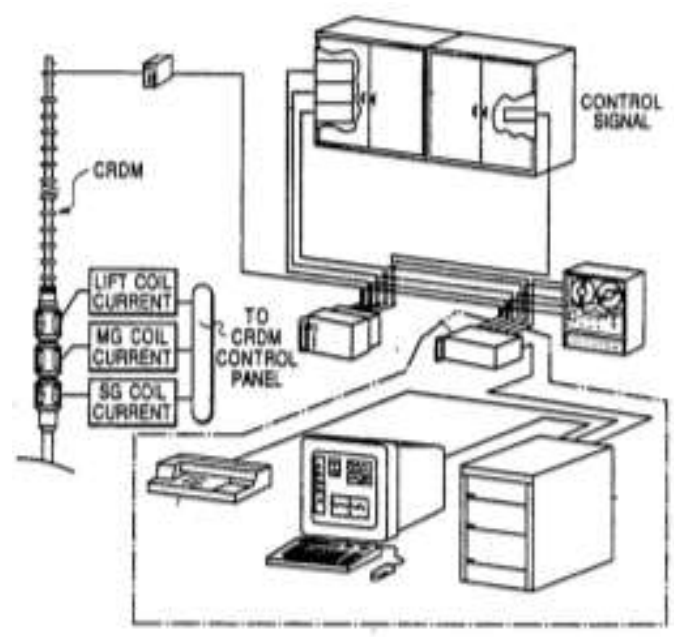

(b)

Fig. 2. (a) The failure distributions of the CRDM, (b) The faults monitoring system for CRDM [22].

In [22] the monitoring technique using noise detection by an accelerometer and by the analysis of electric current of the CRDM coils have been reported as shown in Fig. 2 (b). In further improvement, the monitoring technique which was aimed to replace the electronic ripple detector for the CRDM in PWRs with the software based system was also reported in [23]. This monitoring technique improves the limitation of ripple failure detections by adding a phase failure detection system using wavelet transform. Electric current signal measurement was also applied for step-out error detection [24], accelerated screening test for infant mortality product [21], motor's parameter identification [25] and control [11,26,27]. Although the electric current monitoring has been demonstrated as one of the solutions which has been proposed in magnetized coil-based CRDM, but has not been investigated for the stepper based CRDM specifically in the HTGR plants.

\section{EXPERIMENTAL METHODS}

In this paper, the experiment is focused on stepper motor including the logic generator and power driver as the typical required component in
CRDM of HTGR. The simple CRDM stepping motor based simulator arrangement is sufficient to demonstrate the common faults of typical CRDM components, i.e. logic generator section, power driver section and cable connectors, as mentioned in theory section. In case of other type of faults, such as magnetic damper a gear box, and control rod position detection system, are not considered in this paper due to the unavailability of CRDM HTGR mockup.

The experimental system is developed using a bipolar motor stepper which is driven by a PLC (logic unit) and an H-Bridge circuit (power driver unit) as given in Fig. 3. Note that although the type of stepper motor used here is not specifically designed for the CRDM HTGR, its specifications matching the requirement of a CRDM as well as to demonstrate the condition monitoring methods.

Figure 3 illustrates the arrangement of the CRDM simulator which is combined with the online monitoring system, which is the improved control system reported in [15]. Figure 3 shows that HBridge is employed to drive the motor stepper VEXTA based on the output logic gate of the Programmable Logic Controller (PLC) OMRON CPM1A. In Fig. 3, four channels of PLC with specific addresses generates the logic drive signal while another address are employed to enable the command signal. This logic signals are then switch the H-Bridge circuit to allow sufficient voltage and current to drive the stepper motor. One of the key feature of online monitoring system, a data acquisition system (NI USB 6229) is employed to simultaneously monitor the behavior of output voltage supply of the PLC, the H-Bridge as well as the behavior of stator current of the motor as illustrated in Fig. 3.

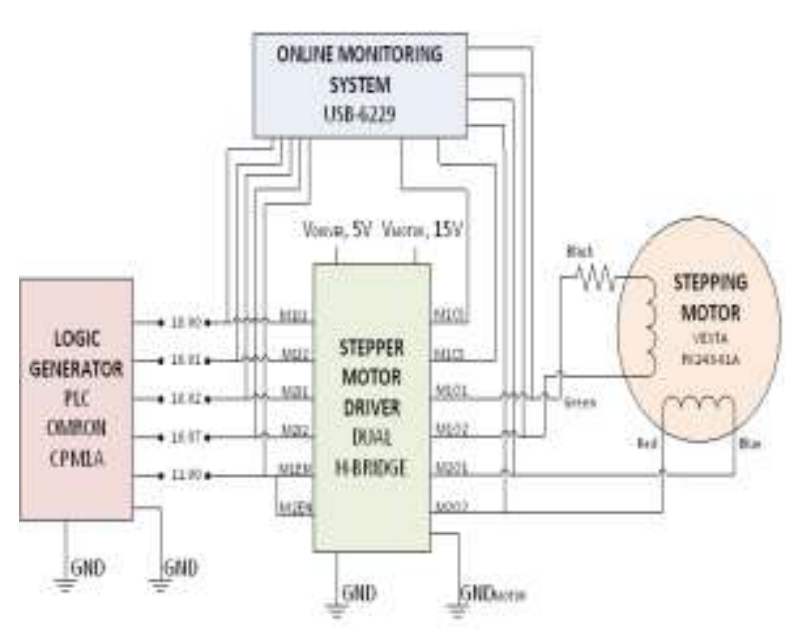

Fig. 3. The arrangement of the monitoring system which are integrated with controlling system, showing an external resistor, $\mathrm{R}_{\mathrm{ext}}$, to simulate degradation due to corrosion. 


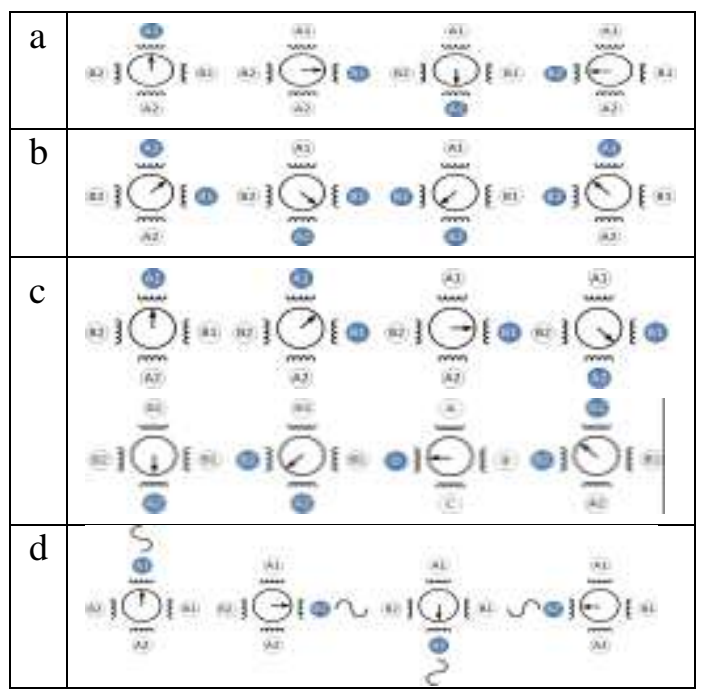

Fig. 4. The stepper motor control techniques using (a) wave drive, (b) full-step, and (c) half-step (d) simple microstep [15].

Various stepper motor logic patterns are generated in the test setup to operate the rotor either clock wise or counter clockwise direction to raise or lower the control rod respectively. Figure 4 illustrates logic patterns for the motor wave drive, full step and half step drive. This logic pattern is needed to understand the nature of the voltage and stator current pattern which is utilized to identify the motor's faults. Figure 4 shows that the voltage supply is applied to activate certain stator poles in sequence. The simplest control methods can be achieved using wave drive technique as illustrated in Fig. 4 (a) (sequence A1-B1-A2-B2). For a smoother rotating torque, full step can be applied as given in Fig. 4 (b) (sequence A1B1-B1A2-A2B2B1A1). In addition, half step control can be done to improve the torque ripple further as illustrated in Fig. 4 (c) (sequence A1- A1B1-B1- B1A2-A2A2B2-B2- B1A1).

The online non-invasive monitoring methods is carried out in the following steps. The online monitoring system acquires output voltage and current signals of the logic generator unit (PLC) and motor driver unit (H-Bridge) simultaneously. These voltage and current signals are investigated to understand the healthy signature of each unit. The fault detection of online monitoring is done by comparing the output voltage pattern of the motor drive unit to the generator unit, called pattern matching. This pattern matching is also synchronized with a standard clock signal to confirm any phase failures. This paper demonstrates this pattern matching with the wave drive, full drive and half drive logic pattern cases both for clockwise and counter clockwise rotation direction. Furthermore, the measured motor stator current and calculated torque can be also examined to confirm the signatures of the stepper motor faults as well as the effect of stepper motor loading which are not easily identified through measured voltage assessment only. The faults due to high-resistance connector between the motor driver and the stepper motor are also simulated using the external resistor as shown in Fig. 3. This simulated fault is employed to demonstrate the applicability as well as the validation of this method in identifying the common fault problems of CRDM as given in the previous section. Detail implementation of this online monitoring for CRDM is discussed in the following section.
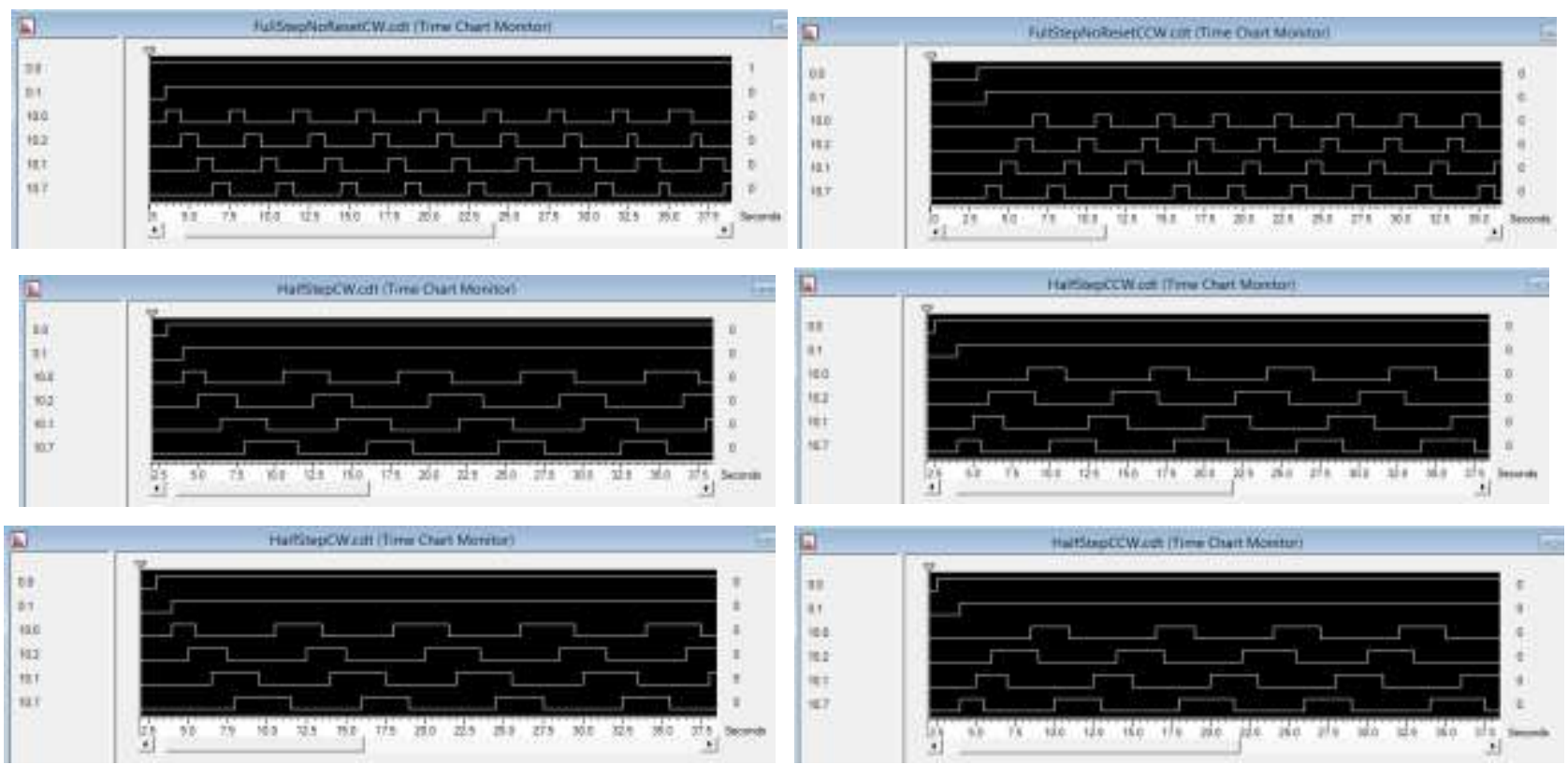

Fig. 5. Timing diagram of the PLC for the wave drive (top row) full step pattern (middle row), and (bottom row) half step drive, the left side is for clockwise and right side column for the counter clockwise rotation 


\section{RESULTS AND DISCUSSION}

The first approach in online noninvasive condition monitoring method is pattern matching between each typical output component of the CRDM HTGR, such as the logic generator unit signal, and the driver unit signal, which is based on comparison at shape and timing of each output block. This technique identifies the performance and the faults in each component of stepping motor, and also their influences on one CRDM component in terms of fault signal classifications. Due to the nature of pattern matching, the monitoring approach requires simultaneously acquisition of the signals from the output channel of each component.

Figure 5 illustrates the control logic patterns of logic generator unit using wave drive (top row), full step (middle row) and half step (bottom row) which are captured using the monitoring system. Noted that the frequency of excitation in each coil is reduced to provide a better visualization in logic transitions.
In Fig.5, the control logic patterns indicate the sequence of each port address either for clockwise (left column) and counter clockwise rotation (right column) for various types of control, i.e. wave drive, full step and half step. For example, in case of wave drive, the port output PLC will activate output address $1000,1002,1001$, and 1007 in sequences which correspond to the activation of coil A1, B1, A2 and B2 respectively as a function of time. In the case of counter clockwise rotation the drive sequence is reversed as $\mathrm{B} 2, \mathrm{~A} 2$, $\mathrm{B} 1$ and $\mathrm{A} 1$.

Figure 6 shows the pattern matching between the output signal of logic generator unit and the output signal of motor driver unit. In this test result, the stepper motor is driven at $5 \mathrm{rpm}$ under three different methods, i.e. the wave drive, full step and half step control. The figure demonstrates that there are no loss of phase between the delivered control logic of the PLC and the generated signal of the motor drive.
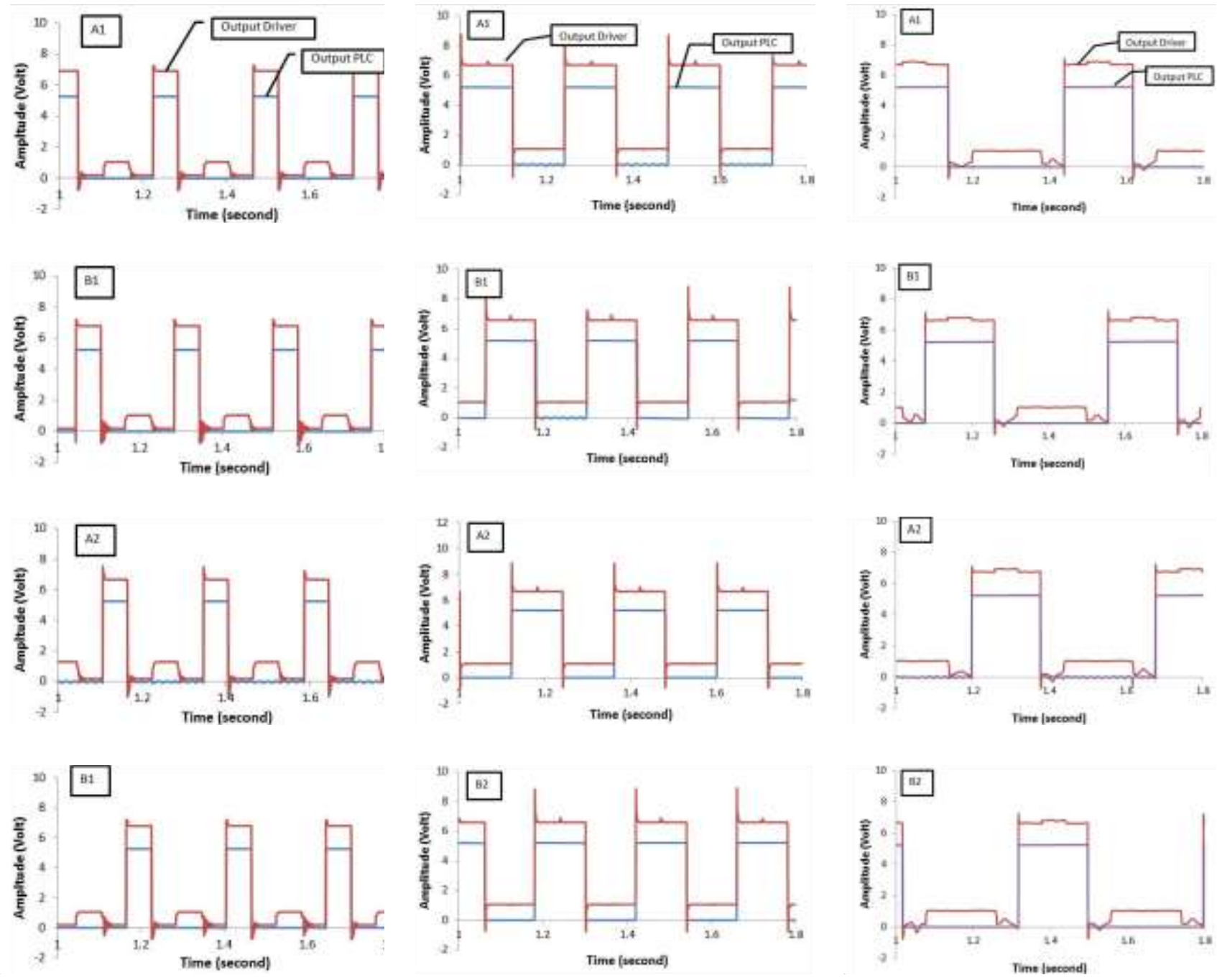

Fig. 6. The voltage timing diagram of the motor for the wave drive (top row) full step pattern (middle row), and (bottom row). 
These results indicate that the logic of PLC can be one of the signature of fault indicator in the form abnormal phase shift sequence. To verify the faults on phase sequence, an independent clock signal which is synchronized with both timing of logic generator and motor driver can also be added.

Another signature of pattern signal, the voltage signals have a small overshoot at the beginning of the step, as well as undershoot followed by a small oscillation at the end of a step, as shown in Fig 6. The over and undershoot voltages are usually produced by the effect of moment inertia force while the rotor moves from one pole position to another. The level of inertia can cause rotor oscillation instead of settling after accelerating from one position to the next. These oscillations can be identified by voltage measurement. Such oscillation can be reduced by a damper or certain load. Hence, this feature can be applied to indicate loading or no loading in the drive for condition monitoring purposes.

Figure 6 also indicates out the possibility to monitor the effect of mutual induction between two active poles. The mutual inductance is recognized from small voltage steps about 1 Volt, (see Fig. 6, left column). Although such voltage is a disturbance in a monitoring system. It is beneficial for the monitoring purposes as a parameter for a healthy signature stepper motor of CRDM with healthy stator windings.

Figure 7 illustrates the stator current monitoring of two stepper motor coils A1-A2 and B1-B2. In case of a wave drive, the generated stator current shows the on-off sequence in each pole pair at 0.9 A. Furthermore, for the full step the stator current monitoring overlaps between coil A1-A2 and B1-B2 (at around $0.9 \mathrm{~A}$ ) because one pole must be activated in each step. In case of the half step operation, the contribution of one coil activation and two coil activations can be differentiated (see Fig. 7). It should be noted that the pattern of current cannot be utilized in pattern matching. However, the information of stator current can be very useful to represent the online torque disturbances or motor faults during the CRDM operations.
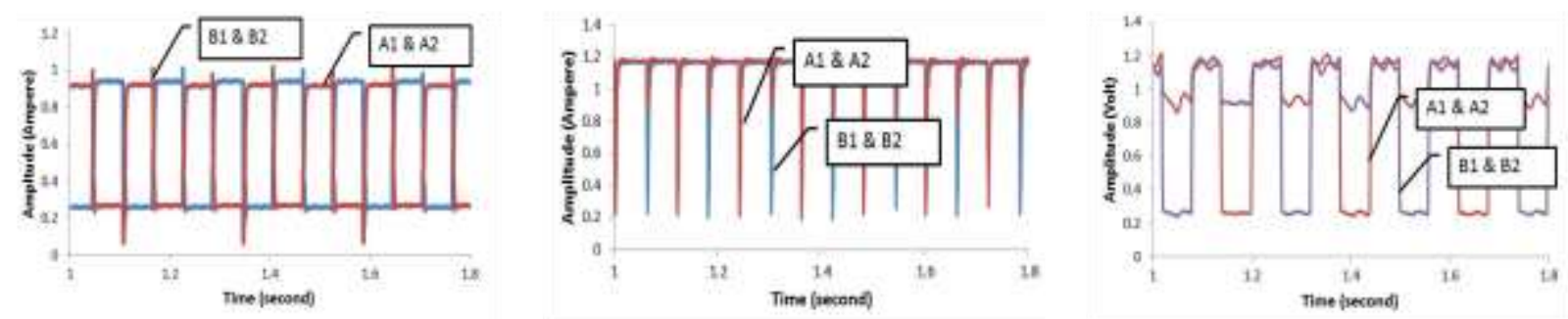

Fig. 7. The stator current timing diagram of the motor for the wave drive (left) full step pattern (middle), and half step drive (right).

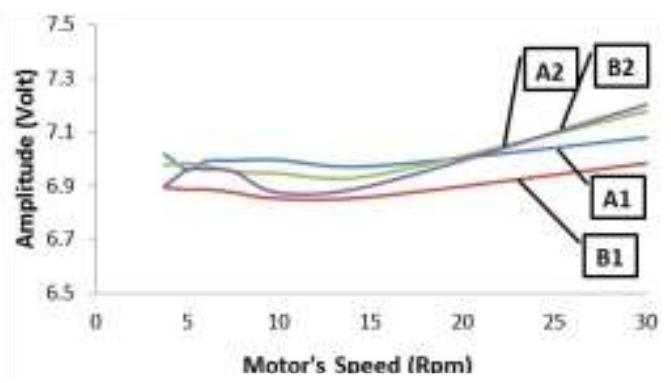

(a)

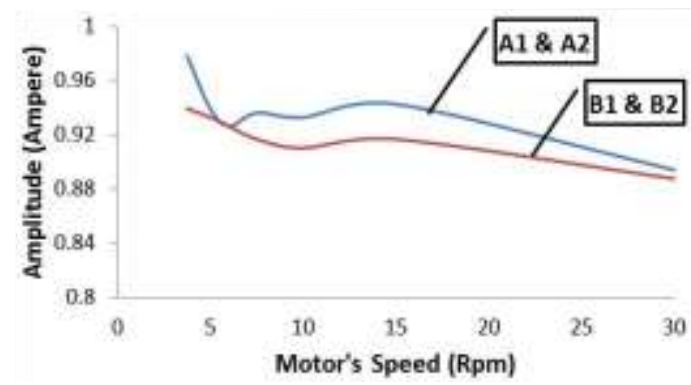

(b)

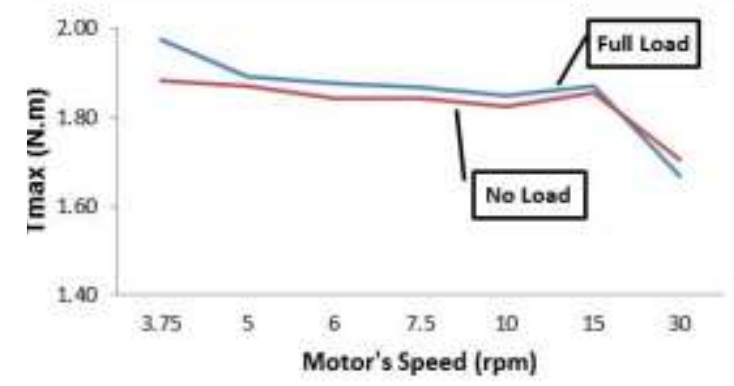

(c)

Fig. 8. The effect of motor's speed to (a) measured voltage (b) measured stator current, and (c) torque. 
The condition monitoring should able to cope with the various speed set points. The speed fluctuations event though is not expected in CRDM HTGR due to the constant acceleration setting, the investigation is required to understand the behavior of measured of voltage and current as a function of speed. The results are illustrated in Fig. 8.

Figure 8 illustrates that the speed variations affect the stator current more than the voltage supply. Note that motor supply voltages increases about $2.4 \%$ while the stator current decreases $6.8 \%$ at a speed of $30 \mathrm{rpm}$. The increase of motor speed means that the maximum loading torque and no loading torque decrease in line with the stator current that creates the magnetic field of the stator as shown in Fig. 8 (c).

The voltage and stator current measurement also show that condition monitoring of CRDM should consider all phases of the stator. The voltage and stator current can be different in each phase due to fabrications, defect during installation, degradation or faults during the operations. As indicated in Fig. 7 the maximum voltage discrepancy of four phases and the stator current are around $3 \%$ and $4 \%$ respectively.

The condition monitoring also demonstrates a detection of high-resistance connectors which can lead to a fault in CRDM. The high-resistance connection in the test system is simulated by an additional resistances in one of stator phase of the output motor drive unit (see Fig. 3).

Figure 9 shows the four phases of voltage and current under the line supply voltage connector problem in phase A1 (using 4 ohms resistor). The stepper motor is controlled to rotate $30 \mathrm{rpm}$. The simulated high-resistance problem reduces the voltage supply in phase A1 as well as the current channel monitoring in A1 \& A2 creating supply voltage and current unbalances.

Figure 9 also shows the rate of increase and reduction in stator current in each step. Note that the
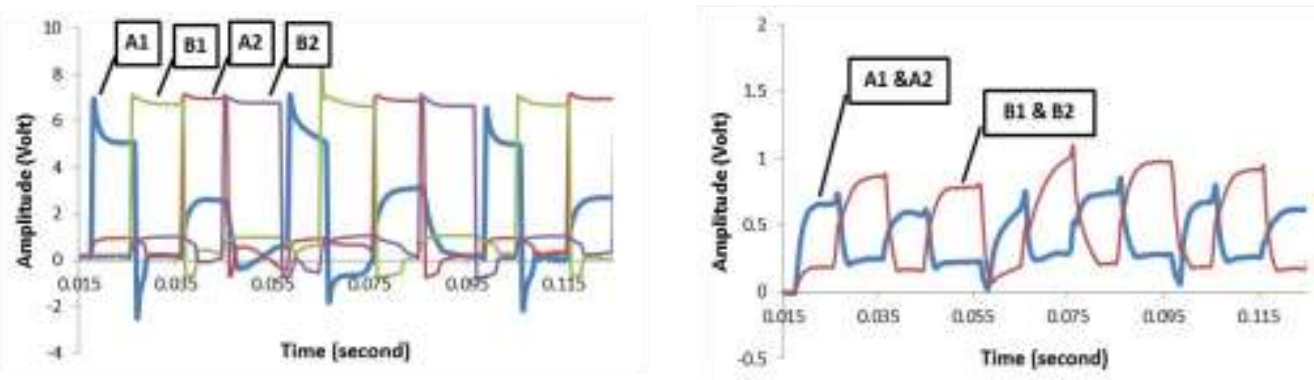

Fig. 9. The voltage timing diagram (left) and stator current (right) monitoring for the wave drive control under single phase degradation (phase A1).

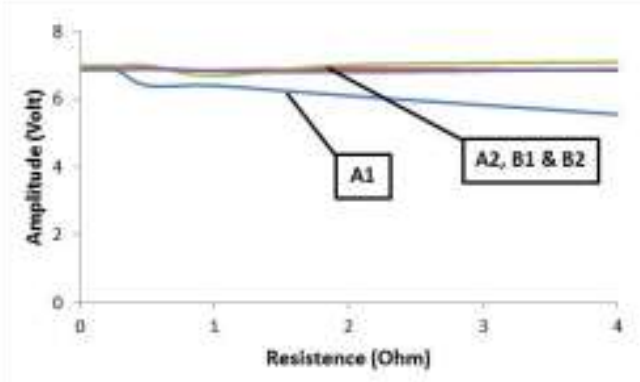

(a)

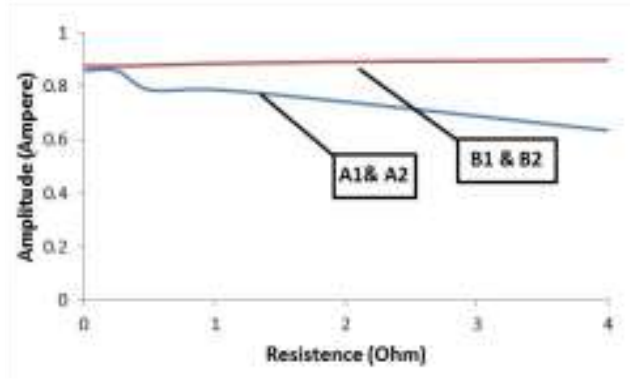

(b)

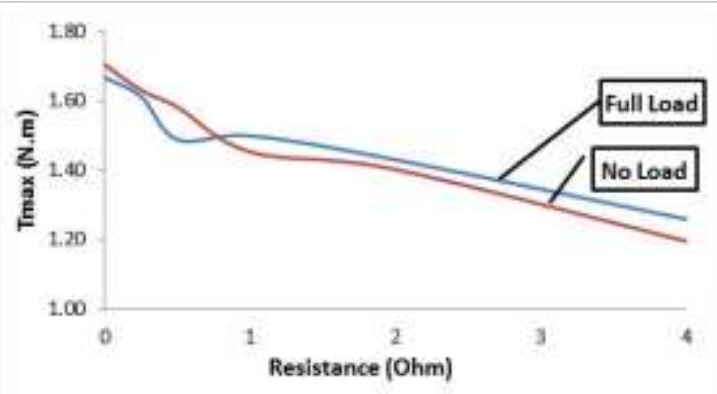

(c)

Fig. 10. The decrease of (a) measured voltage (b) measured current, and (c) torque under single phase degradation (phase A1). 
current variation is affected by the ratio of the inductance and the resistance of the winding $(\mathrm{L} / \mathrm{R})$, known as the time constant of the stepper motor $\left(\mathrm{T}_{0}\right)$. Compare with the healthy stepper motor with a time constant of 1.2 millisecond (for the test motor specification of $8.4 \mathrm{ohm}$ resistance and $10 \mathrm{mH}$ of inductance), additional resistance reduces the time constant, which means that it has sharper transition between each step than the healthy state. Note that such faults reduces the efficiency of the motor.

It can be concluded that 1 ohms extra highresistance reduces the voltage (A1) and current (A1 and A2) for about $13 \%$ and $11 \%$ respectively. The additional resistances affect the current more than the voltage, the smallest detectable severity due to high-resistance (an increase of $2.9 \%$ results in $5.1 \%$ reduction in the stator current).

The high-resistance problem also affects the torque as shown in Fig. 10 (c). The minimum detectable decrease in torque value due to $2.9 \%$ additional high-resistance is about $3.3 \%$. This torque analysis in condition monitoring is necessary to determine the capacity of CRDM to raise or lower control rod under the normal operation as well as variation on the voltage supply. Two factors contribute to the torque reductions. The first factor is the switching time of the stator current. The resultant torque reduces during current commutations between phases. Secondly, the total no load and full load torques of corroded line supply is lower than the healthy line (see Fig. 10 (c) with no additional resistance), due to the reduced stator current which is required to generate magnetic field.

Even though this monitoring technique is capable of identifying the stepper motor problems, however, the method is limited on the electrical related problems. The mechanical related CRDM problems such as the faults in magnetic damper, the gear box, sprocket wheel, worm-gear reducer might not be detected through the voltage and stator current measured signals easily.

\section{CONCLUSION}

It can be concluded that the online noninvasive monitoring technique based on the supply voltage, stator current, and torque monitoring demonstrates an approach which can be applied for a noninvasive condition monitoring technique of CRDM HTGR. The patterns of voltage output signals in each block components of CRDM are compared to determine the healthy and faulty states of the motor. The knowledge of the control pattern from output of the logic generator as well as the generated supply voltage signatures are the key feature for condition monitoring method. The measured stator current and torque are also further signatures to determine the severity and effect of the faults of stepping motor CRDM.

The proposed online non-invasive condition monitoring method successfully identifies the poor connection problem using high-resistance approaches based on measured voltage, stator current which is used also for the torque analysis. It was found that $2.9 \%$ increase in resistance results in $5.1 \%$ and $3.3 \%$ reduction in current and torque values respectively. The method also provide signatures of healthy motor, such as mutual inductions of the motor's winding in voltage and current measurement, the effect of moment inertia, the effect of speed variation, time constant and torque which can be used to identify any abnormalities in the drive.

Future research should consider further complete validation and verification of the method for real CRDM HTGR. In addition, other types of electrical related faults, such as winding motor faults, or rotor faults, as well as the mechanical related faults can be investigated.

\section{ACKNOWLEDGMENT}

The authors acknowledge the support provided by Johan Abdillah Indrapranata and Dwika Saputra during the test phase of the project. The authors are also grateful to the Center for Nuclear Reactor Technology and Safety for the permission granted to use the equipment and facilities.

\section{REFERENCES}

1 L. JaeSeon, J. HyungGil and Y. Young Jung, IEEE Trans. Magn. 50 (2014) 1.

2 Q.-f. Liu and H.-1. Bo, Ann. Nucl. Energy 71 (2014) 237.

3 S.A.M. Shirazi, C. Aghanajafi, S. Sadoughi and N. Sharifloo, Ann. Nucl. Energy 37 (2010) 1659.

4 V. Rajan Babu, R. Veerasamy, S. Patri et al., Nucl. Eng. Des. 240 (2010) 1728.

5 J.H. Lee, S. Kim, Y.-S. Yoo, Y.-G. Cho et al., Nucl. Eng. Des. 300 (2016) 222.

6 W. Yuanqiang, D. Xingzhong, Z. Huizhong et al., Nucl. Eng. Des. 218 (2002) 147. 
7 Y. Tachibana, S. Shiozawa, J. Fukakura, et al., Nucl. Eng. Des. 172 (1997) 93.

8 C. Chen and X. Diao, 21st International Conference on Nuclear Engineering 2 (2013).

9 Z. Dong, Prog. Nucl. Energy 78 (2015) 216.

10 M. Divandari, M. Hashemi-Tilehnoee, M. Khaleghi et al., Nukleonika 59 (2014) 73.

11 S.E. Lyshevski, Energy Conversion and Management 85 (2014) 245.

12 S.H. Khan and A.A. Ivanov, IEEE Trans. Magn. 31 (1995) 2324.

13 R. Kępiński, J. Awrejcewicz and D. Lewandowski, International Journal of Dynamics and Control 3 (2015) 31.

14 K. Balakrishnan, B. Umamaheswari and K. Latha, Industrial Informatics, IEEE Transactions on 9 (2013) 1056.

15 S. Bakhri, Proceedings of National Nuclear Energy Technology Seminar (SENTEN) 2 (2014) 533.

16 M. Mengoni, L. Zarri, Y. Gritli et al., IEEE Trans. Ind. Appl. 51 (2015) 1579.

17 M. Mengoni, L. Zarri, A. Tani et al., IEEE Trans. Power Electron 30 (2015) 4505.
18 P.M.d.l. Barrera, G.R. Bossio and J.A. Solsona, IEEE Trans. Ind. Electron. 61 (2014) 3563.

19 C. Lange, W.H. Fietz and F. Gröner, Fusion Eng. and Des. 88 (2013) 1581.

20 P.M.d.l. Barrera, G.R. Bossio and R. Leidhold, IEEE Trans. Ind. Electron. 62 (2015) 4374.

21 A. Carignano, A. Di Nisio, A.M.L. Lanzolla et al., Measurement 54 (2014) 241.

22 W. Gunther and K. Sullivan, IEEE Trans. Nucl. Sci. 38 (1991) 1760.

23 C. Jong-Min, K. Choon-Kyoug, L. Jong-Moo et al., IEEE Int. Symp. on Ind. Electron. 2 (2006) 1566.

24 S. Moon and D.H. Kim, Mechatronics 24 (2014) 265.

25 R. Delpoux, M. Bodson and T. Floquet, Control Engineering Practice 26 (2014) 178.

26 W. Kim, D. Shin, Y. Lee and C.C. Chung, Mechatronics 35 (2016) 162.

27 Q.F. Qun, J. Xue-Dong and Z. Shi-qing, Procedia Engineering 15 (2011) 2276. 